\title{
Non-Darcy Flow and Heat Transfer in a Porous Insulation with Infiltration
}

Hwa-Chong Tien

Associate Professor, Department of Mechanical and Marine Engineering National Taiwan Ocean University Keelung, Taiwan 202, ROC

Kwang-Sheng Chiang

Graduate student, Department of Mechanical and Marine Engineering National Taiwan Ocean University Keelung, Taiwan 202, ROC

Follow this and additional works at: https://jmstt.ntou.edu.tw/journal

Part of the Mechanical Engineering Commons

\section{Recommended Citation}

Tien, Hwa-Chong and Chiang, Kwang-Sheng (2009) "Non-Darcy Flow and Heat Transfer in a Porous Insulation with Infiltration," Journal of Marine Science and Technology. Vol. 7: Iss. 2, Article 8.

DOI: $10.51400 / 2709-6998.2531$

Available at: https://jmstt.ntou.edu.tw/journal/vol7/iss2/8

This Research Article is brought to you for free and open access by Journal of Marine Science and Technology. It has been accepted for inclusion in Journal of Marine Science and Technology by an authorized editor of Journal of Marine Science and Technology. 


\title{
NON-DARCY FLOW AND HEAT TRANSFER IN A POROUS INSULATION WITH INFILTRATION AND NATURAL CONVECTION
}

\author{
Hwa-Chong Tien* and Kwang-Sheng Chiang**
}

Keywords: natural convection, infiltration, non-Darcian effects.

\section{ABSTRACT}

This work analyzes non-Darcy flow and heat transfer in a vertical slot filled with a porous matrix. The left and right vertical boundaries of the porous system are maintained at higher and lower temperatures, respectively. The horizontal boundaries are assumed to be impermeable and insulated. The vertical boundaries are either completely impermeable, where free convection is induced, or partially permeable in a way to simulate the wall leakage in the building materials, where both air infiltration and free convection exist. The non-Darcy model, which includes the Brinkman and Forchheimer extensions along with the convection terms, is employed to describe the flow in the porous medium. A control-volume-based finite difference scheme is developed to solve the pertinent governing equations along with the boundary conditions. The influence of the locations of the wall leakage is investigated in detail by examining several representative cases. The significance of the non-Darcian effects is shown clearly by comparing the results based on the non-Darcy model with that based on the Darcy model. The enhancement for the heat loss across the porous insulation due to infiltration was found to be case dependent for both Darcy and non-Darcy flows.

\section{INTRODUCTION}

Flow, heat and mass transfer in porous media has been studied extensively. This is due to the increasing need in understanding the complicated transport process for application of diverse fields which include geothermal engineering, building insulation, energy conservation, solid matrix heat exchangers, oil extraction, underground disposal of nuclear waste materials, and many more.

In modeling the flow in porous media, Darcy's law is one of the most popular models. However, it is generally recognized that Darcy's model is valid under the condition that the order of magnitude of the pore

Paper Received September 15, 1999. Revised October 5, 1999. Accepted December 30, 1999. Author for Correspondence: H. C. Tien.

*Associate Professor, Department of Mechanical and Marine Engineering National Taiwan Ocean University Keelung, Taiwan 202, ROC.

**Graduate student, Department of Mechanical and Marine Engineering National Taiwan Ocean University Keelung, Taiwan 202, ROC.
Reynolds number should be less than one. For fluid flow of high velocity and/or porous materials of large pore radius, Forchheimer's modification is suggested by adding the quadratic inertia term to the original Darcy's model. In addition, Brinkman modification accounting for the boundary friction for viscous fluid flowing through porous medium of high permeability was found to be necessary, as pointed out in Ref. [1]. In Ref. [2], a generalized model for describing the flow in porous media was proposed to account for the inertia, the boundary friction and the convective terms. As the permeability of the porous matrix increases, this generalized model approaches the Navier-Stokes equation for pure fluid flow asymptotically.

Building insulation technology is one of the engineering applications related to flow, heat and mass transfer in porous media. References [3, 4] are the pioneering works, which studied both effects of natural convection and infiltration across a vertical porous insulation. It was found in [3, 4] that air infiltration through the wall leakage plays the dominant role in heat transfer. Lauriat and Mesguich [5] investigated both natural convection and radiation effects in a vertical enclosure partially filled with a porous insulation. Darcy's law was envoked in [3-5] for modeling the fluid flow through the porous matrix. Later, Sathe et al. [6] studied natural convection in enclosures containing an insulation with a permeable fluid-porous interface. Brinkman-extended Darcy equation was employed to account for no-slip condition at the wall and the interface. An interesting result was found in [6] which shows that for certain values of the controlling parameters, the heat transfer across the enclosure can be minimized by filling the enclosure only partially with a porous insulation rather than filling it entirely.

Based on the above brief review on the literature regarding building insulation technology, non-Darcy forced and free convection through a vertical slot filled with a porous insulation has not been researched. This constitutes the motive of the present study. All the nonDarcy terms mentioned above, namely, the Forchheimer, the Brinkman, and the convective terms, are included in 
the present work. Four representative opening locations are selected for examining the infiltration effects. The difference between the present results based on the non-Darcy model and those based on the original Darcy model including the results from [3] is demonstrated and discussed.

\section{MATHEMATICAL FORMULATION}

\section{Governing Equations}

The present study is formulated in transient twodimensional natural and forced convection in porous medium. However, the results presented in this work will be based on steady state cases for the sake of simplicity. The common assumptions made in this paper include constant-property fluid, Boussinesq approximation for the buoyancy terms, homogeneous and isotropic porous structure, and local thermal equilibrium. The dimensionless governing equations accounting for the Forchheimer, Brinkman and the convective effects, after invoking the above assumptions, assume the form as follows

$$
\begin{aligned}
& \nabla \bullet \vec{V}=0, \\
& \frac{D a}{\lambda \operatorname{Pr}}\left[\frac{1}{\varepsilon} \frac{\partial \vec{V}}{\partial t}+\frac{1}{\mathcal{E}^{2}}(\vec{V} \bullet \nabla) \vec{V}\right]=-\nabla P+R a^{*} \theta \vec{j}-\vec{V} \\
& -\frac{F s}{\lambda \operatorname{Pr}}|\vec{V}| \vec{V}+D a \nabla^{2} \vec{V}, \\
& \sigma \frac{\partial \theta}{\partial t}+(\vec{V} \bullet \nabla) \theta=\nabla^{2} \theta .
\end{aligned}
$$

The non-dimensional variables in the above equations are defined as

$$
\begin{aligned}
& \vec{V}=\frac{\vec{V}^{*} L}{\alpha_{m}}, \quad P=\frac{P^{*} K}{\alpha_{m} \mu_{f}}, \quad \theta=\frac{T-T_{c}}{\Delta T}, \\
& (x, y)=\frac{\left(x^{*}, y^{*}\right)}{L}, \quad t=\frac{\alpha_{m} t^{*}}{L^{2}} .
\end{aligned}
$$

It is noted that in equations (1)-(3), $D a$ is the Darcy number, $\operatorname{Pr}$ the Prandtl number, $R a^{*}$ Darcy-Rayleigh number, $\varepsilon$ porosity, $\sigma$ the heat capacity ratio, $\lambda$ the ratio of the thermal conductivity of the fluid and the thermal conductivity of the porous material, and $F s$ the Forchheimer constant. In the present work, typical values are chosen for computation: $\lambda=1, \varepsilon=0.4, \sigma=1$ and $\operatorname{Pr}=1$. It should be mentioned that the value of $\sigma$ does not affect the steady-state results. As for $F s$, an empirical equation from [1] is adopted as follows

$$
F s=0.55 \sqrt{D a} \text {. }
$$

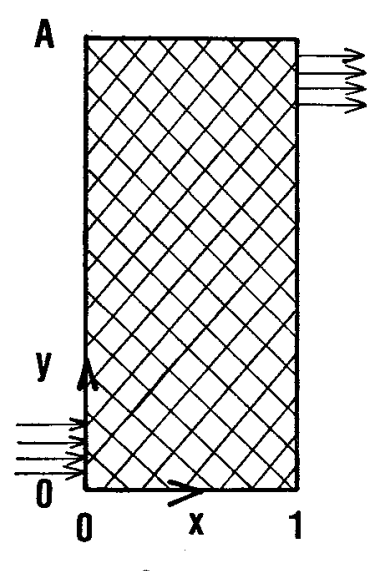

$\underline{\text { Case A }}$

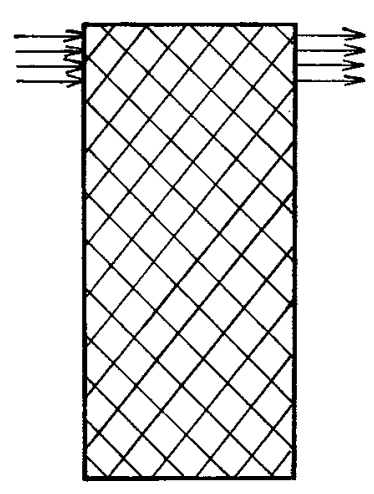

Case C

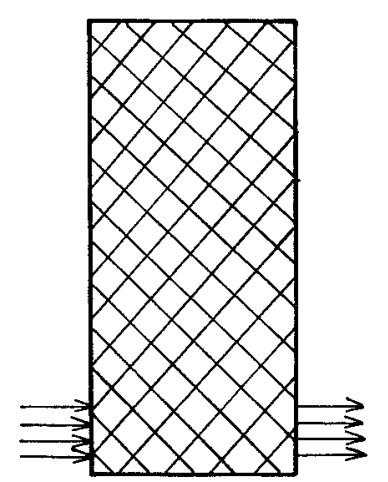

$\underline{\text { Case B }}$

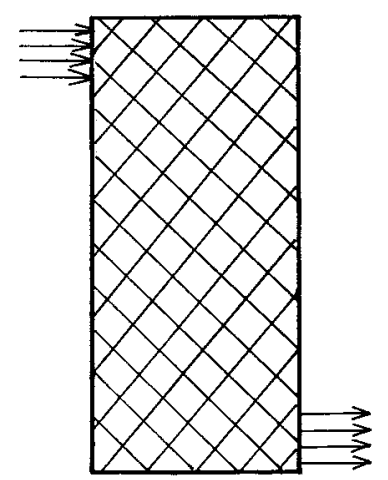

Case D
Fig. 1. Schematic diagram of the present problem.

\section{Boundary Conditions}

The schematic diagram of the present problem is shown in Fig. 1. The left and the right boundaries of the slot are either impermeable or partially permeable. The left boundary is kept at a higher temperature whereas the right boundary is kept at a lower temperature. The top and bottom boundaries are insulated and impermeable. Such configuration can qualitatively simulate the crack or holes in the building insulation. The boundary conditions in dimensionless form are written as

$$
\begin{aligned}
& u=v=0, \frac{\partial \theta}{\partial y}=0 \text { for } 0 \leq x \leq 1, y=0, A \\
& u=v=0, \theta=\theta_{h}=1 \text { for } x=0,0 \leq y \leq A, \\
& u=v=0, \theta=\theta_{c}=0 \text { for } x=1,0 \leq y \leq A
\end{aligned}
$$

The above boundary conditions for the $\mathrm{x}$-component 
velocity are based on impermeable boundaries. For the cases of partially permeable boundaries, the boundary conditions for the $\mathrm{x}$-component velocity for the permeable portion of the wall should be modified as

$$
u=u_{w} \text { on the permeable portion at } 0 \leq y \leq A \text {. }
$$

In equation (7), $u_{w}$ denotes the dimensionless infiltration velocity, which is defined by $u_{w}=u_{w} * / \alpha_{m}$, where $u_{w}{ }^{*}$ denotes the dimensional infiltration velocity. The fluid flowing into the porous system is assumed to be at the dimensionless temperature $\theta_{h}(=1)$ while the fluid flowing out at $\theta_{c}(=0)$. The fluid velocity at the exit is assumed to be the same as the infiltration velocity due to conservation of mass. The locations of the openings are shown in Fig. 1 for representing four typical cases. It should be mentioned that these cases have been studied in Burns et al. [3] and Burns and Tien [4], which are based on the original Darcy flow model. The opening area is set as one eighth of the total area of the vertical boundary.

\section{Initial Condition and Calculation for the Overall Nusselt Number}

The initial condition for $u, v$ and $\theta$ are given by

$$
u=v=0, \theta=\theta_{c}=0 \text { for } t=0 .
$$

The overall Nusselt number at the hot boundary is calculated numerically via the following equation

$$
\overline{N u}=\frac{1}{A} \int_{0}^{A}\left(u \theta-\frac{\partial \theta}{\partial x}\right)_{x=0} d y .
$$

In the above equation, the contribution to the Nusselt number due to infiltration has been included.

\section{METHOD OF SOLUTION}

A control-volume-based finite-difference scheme developed by Patankar [7] is employed to solve the governing equations along with the boundary conditions and the initial condition. The resultant algebraic equations are solved by using a line-by-line iterative method associated with the relaxation parameters. A non-uniform staggered grid system as used in Manol and Lage [8] is adopted, where the mesh size close to the wall is small and then gradually increases to the core region. Such a grid system has been proved to have the advantage of saving computational time and maintaining satisfactory accuracy. The convergence criteria in iteration are stated below

$$
\left|\frac{\phi_{\text {new }}-\phi_{\text {old }}}{\phi_{\text {old }}}\right|<T o l \text {, }
$$

where $\phi_{\text {new }}$ and $\phi_{\text {old }}$ denote one of the main variables $u$, $v$ and $\theta$ and the subscripts 'new' and 'old' denote the values corresponding to the new iteration and old iteration, respectively. The value for the tolerance Tol is set as $10^{-6}$. Since the present work is based on the transient formulation, the numerical computation continues until the relative difference between the overall Nusselt numbers for two consecutive time steps is less than $10^{-5}$; that is, the steady state is assumed to have been achieved.

\section{RESULTS AND DISCUSSION}

\section{Results for a Fundamental Case and a Comparison}

The objective of the present work is to study the non-Darcy convective flow across the porous insulation. Several checks have been made in order to verify the accuracy of the numerical results. First, for the classical case of impermeable vertical boundaries and aspect ratio of one, the present results based on both Darcy and non-Darcy flow models are presented in Table 1 , in comparison with the results of Lauriat and Prasad [9] which is based on the non-Darcy model. It can be seen clearly that the agreement between the present results based on the non-Darcy model and the results of Lauriat and Prasad [9] is good for low Darcy-Rayleigh numbers, and acceptable for high Darcy-Rayleigh numbers. Similar trend was found for the thermal and fluid fields. As expected, the Nusselt number predicted by the original Darcy flow model is higher than that based on the nonDarcy flow model. The difference for the Nusselt numbers between the Darcy and non-Darcy models increases as the Darcy-Rayleigh number increases. Moreover, for the same Darcy-Rayleigh number, larger Darcy numbers yield larger difference in the Nusselt numbers between the Darcy and non-Darcy models than smaller Darcy numbers.

\section{Results based on the Main Configurations}

Next, attention is turned to the main configurations of the present study. As mentioned in the section of "Introduction", four representative configurations corresponding to different locations of wall openings are considered in the present work. For the remaining part of this section, the aspect ratio is fixed and chosen as 10 . The dimensional infiltration velocity $u_{w}$ is taken as 5 except in Fig. 6 where $u_{w}$ is taken as 15 . The fundamental case of completely impermeable boundaries is also considered for the sake of comparison. Before presenting the results based on the main configurations, the dependence of accuracy on grids is examined. By refining the mesh size from $4 \times 40$ to $8 \times$ 
Table 1. A comparison for the overall Nusselt numbers from the present study based on both the Darcy and the non-Darcy flow models with that from Lauriat and Prasad [9], which is based on the non-Darcy flow model, for $A=1, \lambda=1$ and $\varepsilon=0.4$.

\begin{tabular}{|c|c|c|c|c|c|}
\hline \multirow[t]{2}{*}{ Models } & \multirow[t]{2}{*}{$R a^{*}$} & \multirow[t]{2}{*}{$\mathrm{Da}$} & \multirow[t]{2}{*}{ Lauriat \& Prased } & \multicolumn{2}{|c|}{ Present study } \\
\hline & & & & non-Darcy & Darcy \\
\hline \multirow[t]{4}{*}{ Forchheimer } & 100 & $1.00 \mathrm{E}-06$ & 3.07 & 3.074 & 3.099 \\
\hline & 1000 & $1.00 \mathrm{E}-06$ & 12.8 & 13.01 & 13.53 \\
\hline & 5000 & $1.00 \mathrm{E}-06$ & 26.15 & 26.09 & 35.64 \\
\hline & 5000 & $1.00 \mathrm{E}-04$ & 17.2 & 16.8 & 35.64 \\
\hline \multirow[t]{4}{*}{ Brinkman } & 100 & $1.00 \mathrm{E}-06$ & 3.06 & 3.082 & 3.099 \\
\hline & 1000 & $1.00 \mathrm{E}-06$ & 13.22 & 13.25 & 13.53 \\
\hline & 5000 & $1.00 \mathrm{E}-06$ & 31.5 & 32.1 & 35.64 \\
\hline & 5000 & $1.00 \mathrm{E}-04$ & 20.85 & 19.85 & 35.64 \\
\hline \multirow{2}{*}{$\begin{array}{l}\text { Forchheimer, Brink- } \\
\text { man \& conv. terms }\end{array}$} & 10000 & $1.00 \mathrm{E}-08$ & 44.3 & 45.4 & 51.97 \\
\hline & 10000 & $1.00 \mathrm{E}-04$ & 18.4 & 17.89 & 51.97 \\
\hline
\end{tabular}

Table 2. Overall Nusselt numbers from the present study based on both the Darcy and the non-Darcy flow models for $A=10, \lambda=1$, $\varepsilon=0.4, u_{w}=5,15$, and $D a=10^{-5}, 10^{-6}$. Also shown are the results from Burns et al. [3], which is based on the Darcy flow model.

\begin{tabular}{rlllclccc}
\hline$u_{w}$ & $R a^{*}$ & \multicolumn{1}{c}{$D a$} & models & Impermeable & Case A & Case B & Case C & Case D \\
\hline 5 & 100 & N/A & Burns et al. & 1.7 & 1.97 & 1.75 & 2.25 & 1.98 \\
5 & 100 & N/A & Darcy & 1.656 & 2.021 & 1.796 & 2.25 & 1.985 \\
5 & 100 & $1.00 \mathrm{E}-06$ & non-Darcy & 1.642 & 1.992 & 1.772 & 2.217 & 1.958 \\
5 & 100 & $1.00 \mathrm{E}-05$ & non-Darcy & 1.614 & 1.962 & 1.748 & 2.189 & 1.931 \\
5 & 500 & N/A & Darcy & 3.804 & 4.217 & 3.943 & 4.371 & 4.083 \\
5 & 500 & $1.00 \mathrm{E}-06$ & non-Darcy & 3.642 & 4.048 & 3.776 & 4.211 & 3.924 \\
5 & 500 & $1.00 \mathrm{E}-05$ & non-Darcy & 3.396 & 3.793 & 3.525 & 3.969 & 3.681 \\
15 & 100 & N/A & Darcy & 1.656 & 2.781 & 2.483 & 3.474 & 2.712 \\
15 & 100 & $1.00 \mathrm{E}-06$ & non-Darcy & 1.642 & 2.766 & 2.468 & 3.459 & 2.699 \\
15 & 500 & N/A & Darcy & 3.804 & 5.006 & 4.218 & 5.559 & 4.651 \\
15 & 500 & $1.00 \mathrm{E}-06$ & non-Darcy & 3.642 & 4.82 & 4.053 & 5.406 & 4.492 \\
\hline
\end{tabular}

80 and then to $16 \times 160$, it was found that the difference for the results of the Nusselt number based on the grid of $8 \times 80$ and that based on the grid of $16 \times 160$ is quite small. The numerical results hereinafter are based on the grid of $8 \times 80$ to save the computational time and maintain reasonable accuracy.

Next, the present results based on the original Darcy flow model for the overall Nusselt number are compared with that from Burns et al. [3] for $u_{w}=5$ and $R a^{*}=100$, as shown in Table 2. It can be seen again that good agreement is achieved. It is also noted that the enhancement of heat transfer due to infiltration is obviously case-dependent. For example, case B renders the Nusselt number only slightly higher than that for the case of completely impermeable boundaries. This is because in case $\mathrm{B}$, the direction of flow due to infiltration is opposite to the buoyancy-induced flow. For illustration, the streamlines and isotherms based on the Darcy flow model are depicted in Fig. 2, where Fig. 2 (a)-(c) correspond to, respectively, the case of completely impermeable boundaries, case B, and case $C$. The streamlines and isotherms for cases A and D are not shown in Figs. 2-6 for brevity. It can be seen from Fig. 2 that the flow field for each case is quantitatively and qualitatively different from each other due to different opening locations. Although the thermal fields for these cases look similar, the difference among them is still visible due to the influence of the flow fields. It should be pointed out that in most cases such as A, B, and D, the overall Nusselt number, as shown in Table 2, is not simply equal to the Nusselt number due to pure natural convection (1.656) plus the contribution due to infiltration (0.625), except for case $\mathrm{C}$, which satisfies the equality approximately.

The effect of the buoyancy force is examined by increasing The Darcy-Rayleigh number from 100 to 500 , which is about the higher end encountered in the application of building insulation technology. Fig. 3 is 

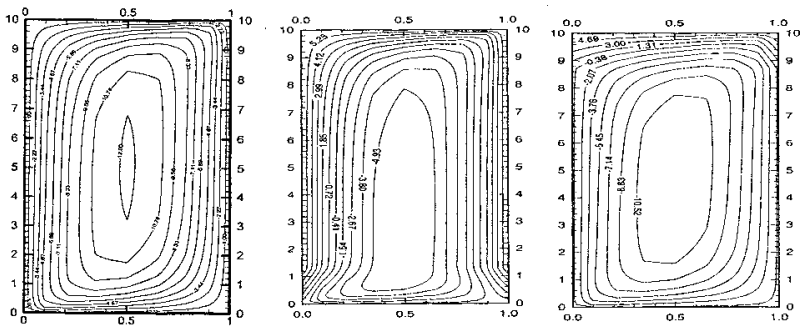

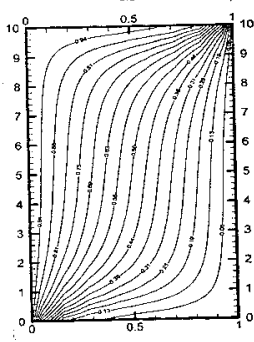

(a)

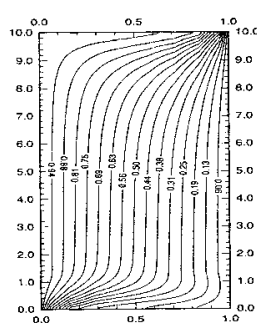

(b)

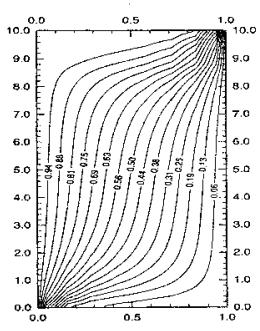

(c)
Fig. 2. Streamlines and isotherms based on the Darcy flow model, $A=10$, $R a^{*}=100, u_{w}=5$, (a) impermeable boundaries, (b) case B, and (c) case C.
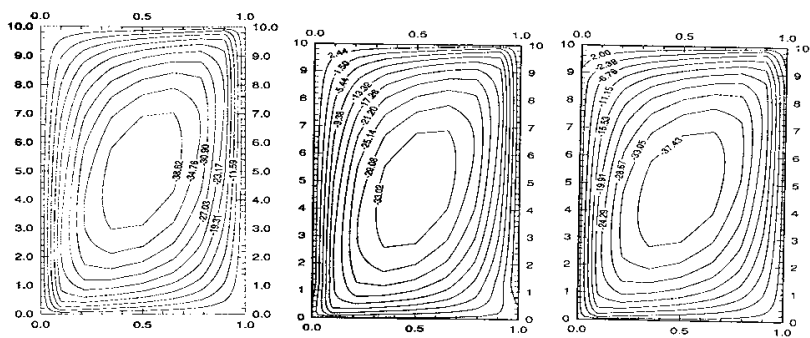

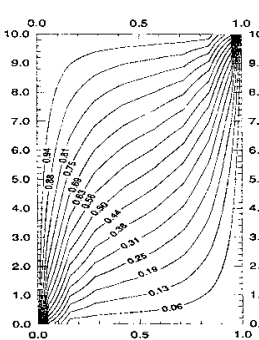

(a)

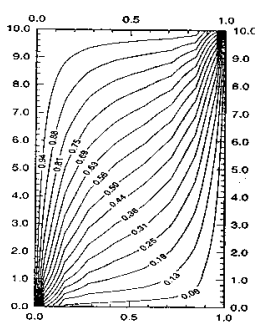

(b)

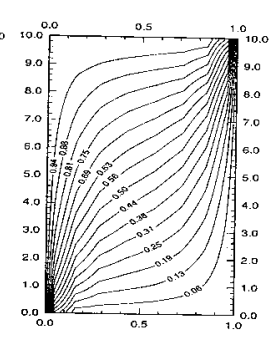

(c)
Fig. 3. Streamlines and isotherms based on the Darcy flow model, $A=10$, $R a^{*}=500, u_{w}=5$, (a) impermeable boundaries, (b) case B, and (c) case $\mathrm{C}$.

based on the Darcy model and $R a^{*}=500$. By observing Table 2 and also comparing Fig. 3 with Fig. 2, it can be found that an increase in the Darcy-Rayleigh number enhances the fluid and the thermal fields and an increase in Nusselt number for all cases. Again, the Nusselt numbers for most cases are not simply equal to the Nusselt number due to natural convection (3.804) plus the contribution due to infiltration $(0.625)$, except for case C.
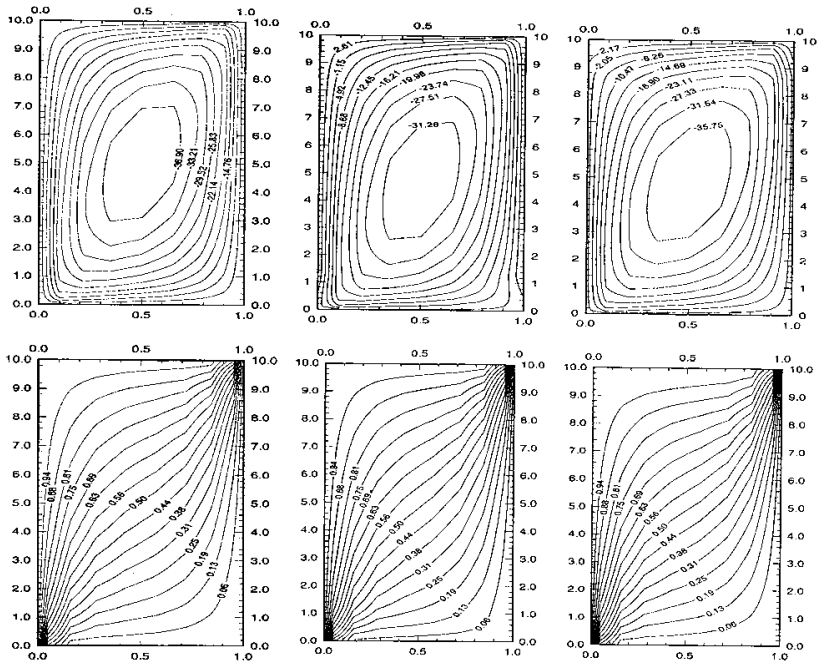

(a)

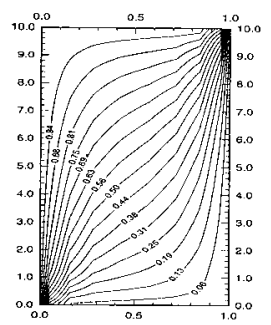

(b)

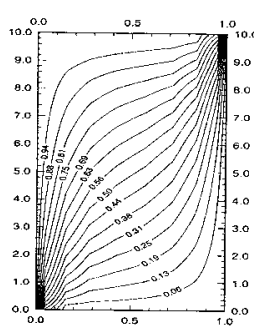

Fig. 4. Streamlines and isotherms based on the non-Darcy flow model, $A$ $=10, \lambda=1, \varepsilon=0.4, R a^{*}=500, D a=10^{-6}, u_{w}=5$, (a) impermeable boundaries, (b) case B, and (c) case $\mathrm{C}$.
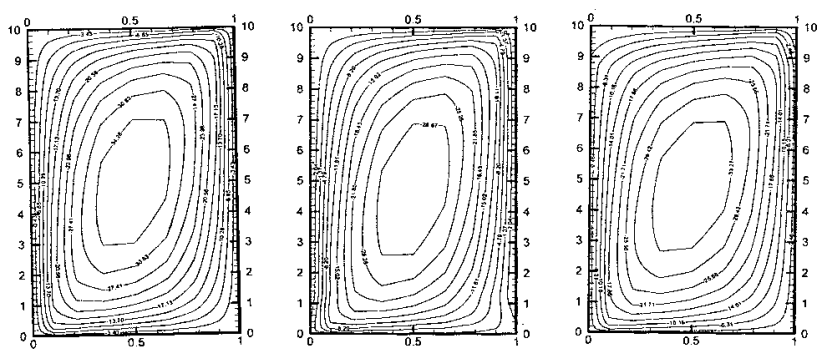

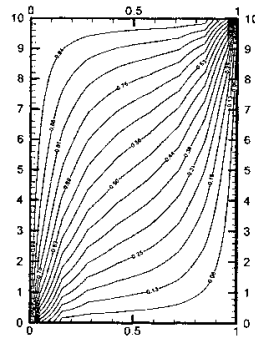

(a)

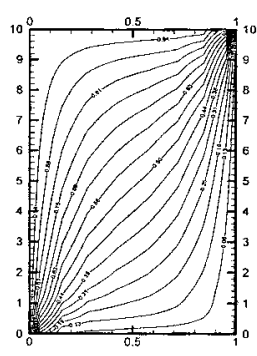

(b)

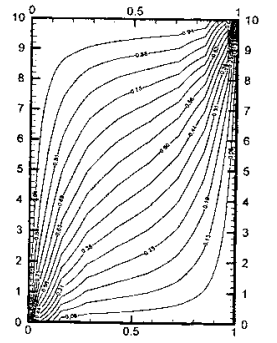

(c)

Fig. 5. Streamlines and isotherms based on the non-Darcy flow model, $A$ $=10, \lambda=1, \varepsilon=0.4, R a^{*}=500, D a=10^{-5}, u_{w}=5$, (a) impermeable boundaries, (b) case B, and (c) case $\mathrm{C}$.

As for the non-Darcian effect, it can be better examined from two viewpoints. First, it is expected that for the low Darcy-Rayleigh numbers, such as $R a^{*}=100$, the difference for the flow and thermal fields and the Nusselt number between the Darcy and non-Darcy models is negligible as shown in Table 2. However, as the Darcy-Rayleigh number increases, the non-Darcian effect increases accordingly. It should be mentioned that 

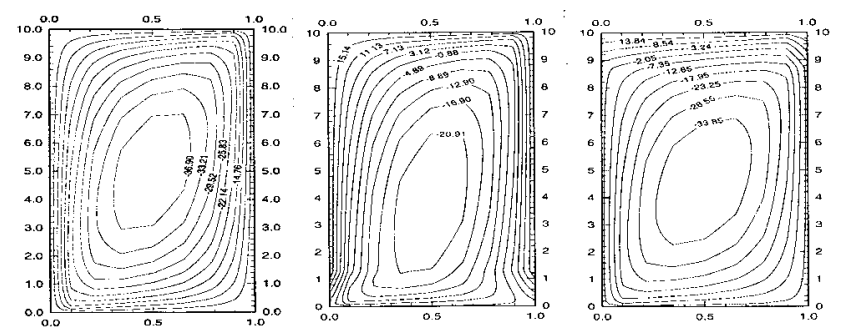

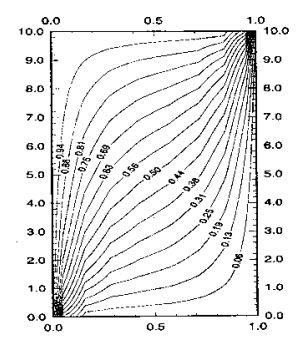

(a)

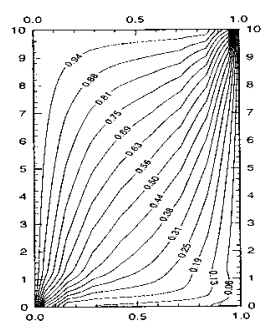

(b)

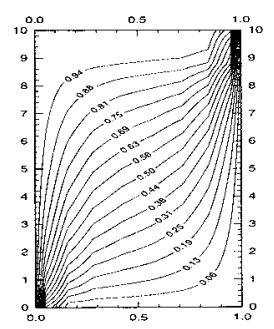

(c)
Fig. 6. Streamlines and isotherms based on the non-Darcy flow model, $A$ $=10, \lambda=1, \varepsilon=0.4, R a^{*}=500, D a=10^{-6}, u_{w}=15$, (a) impermeable boundaries, (b) case B, and (c) case $\mathrm{C}$.

the non-Darcy model in Table 2 includes the Brinkman, Forchheimer, and convection terms. Through the comparison between Fig. 4, which is based on the non-Darcy model, $R a^{*}=500, D a=10^{-6}$, and Fig. 3, which is based on Darcy model with the same $R a^{*}$, it can be seen that the non-Darcy flow fields are weaker than the Darcy flow fields. Moreover, under the condition of the same Darcy-Rayleigh number, the non-Darcian effect is more prominent for higher Darcy numbers as shown in Fig. 5, which is based on the non-Darcy model with $\mathrm{Ra}^{*}=500$ while $D a=10^{-5}$. By comparing Fig. 5 with Fig. 4, it can be seen the fluid fields predicted for $D a=10^{-5}$ is weaker than that predicted for $D a=10^{-6}$ for all cases. This is because for the same Darcy-Rayleigh number $R a^{*}$, higher Darcy number $D a$ means smaller fluid Rayleigh number $R a$ and hence weaker buoyancy force is generated. The results of the Nusselt number in Table 2 also clearly show the trend. It is noted that the magnitudes of the Nusselt numbers based on the non-Darcy model for these four cases are in the same order as that based on the Darcy flow model. Finally, the effect of infiltration velocity is examined by increasing the dimensionless infiltration velocity from 5 to 15 , while $R a^{*}$ and Da are fixed. Figure 6 shows the results of the streamlines and isotherms based on the non-Darcy model for $u_{w}=15$, $R a^{*}=500$ and $D a=10^{-6}$. By comparing Fig. 6 with Fig. 4 , it can be seen that an increase in the infiltration velocity not only enhances the flow field but also alters the thermal field. As expected, the Nusselt number increases as the infiltration velocity increases, as shown in Table 2. It is noted that the variation of the infiltra- tion velocity does not cause any change for the case of completely impermeable boundaries. Also, the increase in the Nusselt number due to the increase in the infiltration velocity is found to be case dependent; in the order of the magnitude of increase, these are case $C$ (1.195), case A (0.772), case D (0.568) and case B (0.277). It is noted that the above increments in the Nusselt number, say for case $C(1.195)$, are obtained by taking the Nusselt number for each case for $u_{w}=15, R a^{*}=500$ and $D a=10^{-6}$, which is 5.406 for case C, minus the Nusselt number for the same case for $u_{w}=5$, which is 4.211 . It should be mentioned that the influence of the infiltration velocity on the overall Nusselt number is similar for the Darcy flow. One more thing should be pointed out that the problem studied in this work can be regarded as a problem of infiltration (forced convection) and free convection. A dimensionless parameter characterizing the problem of mixed convection is defined by $G r / R e^{2}$ or $R a^{*} / R e^{2}$, where $G r$ is the Grashof number defined as $G r=R a^{*} / P r$. If the length of the slot $L$ is given by $0.1 \mathrm{~m}$, then the dimensional infiltration velocity $u_{w}{ }^{*}$ can be found to have the values of $1.4 \times 10^{-3} \mathrm{~m} /$ $\mathrm{s}$ and $4.2 \times 10^{-3} \mathrm{~m} / \mathrm{s}$ for $u_{w}=5$ and 15 , respectively. This in turn gives the value of $R a^{*} / R e^{2}$ ranging from 0.14 to 6.25 approximately. Within such range, both effects of infiltration (forced convection) and free convection are important. This fact also helps us explain the reason why for most cases the overall Nusselt number is not directly equal to the Nusselt number due to infiltration plus that due to natural convection.

\section{CONCLUSIONS}

This paper investigates non-Darcy flow and heat transfer in a porous matrix accounting for both infiltration and free convection effects. The non-Darcy flow model includes Brinkman, Forchheimer and convection terms. The flow field and heat transfer based on Darcy model is also examined for comparison. The nonDarcian effect was found to be negligible for low DarcyRayleigh numbers while they become more pronounced for medium and large Darcy-Rayleigh numbers. For the same Darcy-Rayleigh number, the flow field and heat transfer is depressed as the Darcy number increases. Depending on the locations of the wall openings, the overall Nusselt number is not simply equal to the Nusselt number due to infiltration plus that due to free convection. The increase in the Nusselt number due to the increase in the infiltration velocity is case dependent.

\section{NOMENCLATURE}

aspect ratio, $H / L$

$b$ inertia constant [m] 
$c \quad$ specific heat $[\mathrm{J} / \mathrm{kg}-\mathrm{K}]$

Da Darcy number, $K / L^{2}$

Fs Forchheimer constant, $b / L$

$\vec{j} \quad$ unit vector in the positive y-direction

$H \quad$ height of the slot [m]

$K \quad$ permeability $\left[\mathrm{m}^{2}\right]$

$k_{m} \quad$ thermal conductivity $[\mathrm{W} / \mathrm{m}-\mathrm{K}]$

$L^{m} \quad$ length of the slot [m]

$\overline{N u}$ overall Nusselt number, defined in equation (9)

$P \quad$ pressure

$P r \quad$ Prandtl number of the fluid

$R a$ Rayleigh number of the fluid, $g \beta L^{3} \Delta T / v_{f} \alpha_{f}$

$R a^{*}$ Darcy-Rayleigh number, $g \beta K L \Delta \mathrm{T} / v_{f} \alpha_{m}=\lambda D a R a$

$t$ dimensionless time

$T$ Temperature [K]

$\Delta T$ temperature difference, $T_{h}-T_{c}[\mathrm{~K}]$

$u$ dimensionless velocity in $\mathrm{x}$-direction

$u_{w}$ dimensionless infiltration velocity at the openings

$v$ dimensionless velocity in y-direction

$\vec{V}$ dimensionless velocity vector

$x$, $y$ dimensionless coordinates

\section{Greek Symbols}

$\alpha_{f} \quad$ thermal diffusivity of the fluid $\left[\mathrm{m}^{2} / \mathrm{s}\right]$

$\alpha_{m}$ thermal diffusivity of the porous medium, $k_{m}(\rho c)_{\mathrm{f}}$ $\left[\mathrm{m}^{2} / \mathrm{s}\right]$

$\varepsilon \quad$ porosity

$\lambda$ thermal conductivity ratio, $k_{f} / k_{m}$

$\mu \quad$ dynamic viscosity of the fluid $[\mathrm{kg} / \mathrm{m}-\mathrm{s}]$

$v \quad$ kinematic viscosity of the fluid $\left[\mathrm{m}^{2} / \mathrm{s}\right]$

$\theta$ dimensionless temperature

$\rho$ density $\left[\mathrm{kg} / \mathrm{m}^{3}\right]$

$\sigma \quad$ heat capacity ratio, $\left[\varepsilon(\rho c)_{f}+(1-\varepsilon)(\rho c)_{\mathrm{s}}\right] /(\rho c)_{f}$

\section{Subscripts}

c cold boundary

$f \quad$ fluid

$h$ hot boundary

$m$ fluid saturated porous medium

$s \quad$ solid

\section{Superscripts}

* dimensional quantities

\section{REFERENCES}

1. Bejan, A., "Convective Heat Transfer in Porous Media," in Chap. 16 from Handbook of Single-Phase Convective Heat Transfer, S. Kakac, R. K. Shah and W. Aung, eds., John Wiley and Sons, New York, pp. 16.116.34, (1987).
2. Vafai, K. and Tien, C. L., "Boundary and Inertia Effects on Flow and Heat Transfer in Porous Media," Int. J. Heat and Mass Transfer, Vol. 24, pp. 195-203, (1981).

3. Burns, P. J., Chow, L. C. and Tien, C. L., "Convection in a Vertical Slot Filled with Porous Insulation," Int. J. Heat and Mass Transfer, Vol. 20, pp. 919-926, (1977).

4. Burns, P. J. and Tien, C. L., "Effects of Infiltration on Heat Transfer through Vertical Slot Insulation," In Energy Conservation in Heating, Cooling, and Ventilating Buildings, C. J. Hoogendoorn and N. H. Afgan, eds., Vol. 1, pp. 93-105, Hemisphere, New York, (1978).

5. Lauriat, G. and Mesguich, F., "Natural Convection and Radiation in an Enclosure Partially Filled with a Porous Insulation," ASME 84-WA/HT-101, (1984).

6. Sathe, S. B., Lin, W. Q. and Tong, T. W., "Natural Convection in Enclosures Containing an Insulation with a Permeable Fluid-Porous Interface," Int. J. Heat Fluid Flow, Vol. 9, pp. 389-395, (1988).

7. Patankar, S. V., Numerical Heat Transfer and Fluid Flow, Hemisphere, Washington, D. C., (1980).

8. Manole, D. M. and Lage, J. L., "The Inertia Effect on the Natural Convection Flow within a Fluid-Saturated Porous Medium," Int. J. Heat and Fluid Flow, Vol. 14, pp. 376-384, (1993).

9. Lauriat, G. and Prasad, V., "Non-Darcian Effects on Natural Convection in a Vertical Porous Enclosure," Int. J. Heat and Mass Transfer, Vol. 32, pp. 2135-2148, (1989).

\section{孔狀材料含渗透與自然對流非達西 模式之解析}

$$
\text { 田華忠蔣光聲 }
$$

國立台灣海洋大學機械與輪機工程學系

$$
\text { 摘 要 }
$$

本文採非達西模式對直立孔質内流與熱傳作解 析。孔質系統之左右邊界分別爲高温和低温, 上下邊 界則爲絕熱及不可渗透。垂直邊界或爲不可渗透, 或 爲部份可滲透以模擬建築物牆有開口情況。前者僅須 考虑自然對流, 後者則須加上空氣渗透效應。本文中 非達西模式包括Brinkman、Forchheimer及對流等 項, 採控制體積差分法將描述問題之統御方程式及邊 界條件離散化後求解。本文藉由四個具代表性的例子 以探討開口位置之影響, 並將非達西模式所得之結果 與達西模式所得之結果比較以探討非達西效應。本文 發現不論是達西流或是非達西流, 由於空氣渗透引起 熱傳率之增加因例子不同而有所差異。 